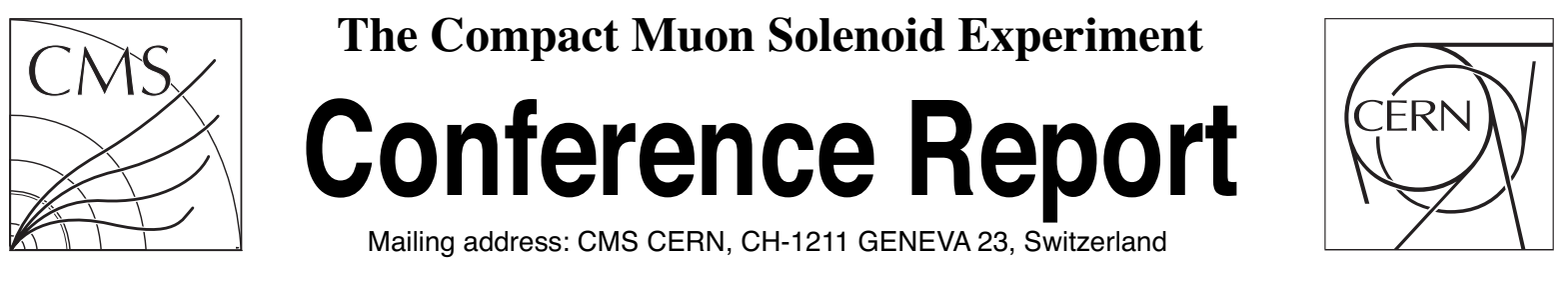

08 December 2017 (v4, 14 December 2017)

\title{
The CMS High Granularity Calorimeter for the High Luminosity LHC
}

Jean-Baptiste Sauvan for the CMS Collaboration

\begin{abstract}
The High Luminosity LHC (HL-LHC) will integrate 10 times more luminosity than the LHC, posing significant challenges for radiation tolerance and event pileup on detectors, especially for forward calorimetry, and hallmarks the issue for future colliders. As part of its HL-LHC upgrade program, the CMS collaboration is designing a High Granularity Calorimeter to replace the existing endcap calorimeters. It features unprecedented transverse and longitudinal segmentation for both electromagnetic (ECAL) and hadronic (HCAL) compartments. This will facilitate particle-flow calorimetry, where the fine structure of showers can be measured and used to enhance pileup rejection and particle identification, whilst still achieving good energy resolution. The ECAL and a large fraction of HCAL will be based on hexagonal silicon sensors of $0.5-1 \mathrm{~cm}^{2}$ cell size, with the remainder of the HCAL based on highly-segmented scintillators with silicon photomultiplier (SiPM) readout. The intrinsic high-precision timing capabilities of the silicon sensors will add an extra dimension to event reconstruction, especially in terms of pileup rejection.
\end{abstract}




\section{The CMS High Granularity Calorimeter for the High Luminosity LHC}

\section{J.-B. Sauvan on behalf of the CMS Collaboration}

Laboratoire Leprince-Ringuet, CNRS/École Polytechnique

E-mail: jean-baptiste.sauvan@cern.ch

Abstract: The High Luminosity LHC (HL-LHC) will integrate 10 times more luminosity than the LHC, posing significant challenges for radiation tolerance and event pileup on detectors, especially for forward calorimetry, and hallmarks the issue for future colliders. As part of its HL-LHC upgrade program, the CMS collaboration is designing a High Granularity Calorimeter to replace the existing endcap calorimeters. It features unprecedented transverse and longitudinal segmentation for both electromagnetic (ECAL) and hadronic (HCAL) compartments. This will facilitate particle-flow calorimetry, where the fine structure of showers can be measured and used to enhance pileup rejection and particle identification, whilst still achieving good energy resolution. The ECAL and a large fraction of HCAL will be based on hexagonal silicon sensors of $0.5-1 \mathrm{~cm}^{2}$ cell size, with the remainder of the HCAL based on highly-segmented scintillators with silicon photomultiplier (SiPM) readout. The intrinsic high-precision timing capabilities of the silicon sensors will add an extra dimension to event reconstruction, especially in terms of pileup rejection.

Keywords: Calorimeters, Radiation-hard detectors, Performance of High Energy Physics Detectors 


\section{Contents}

1 Introduction 1

2 HGCAL design overview 2

3 Reconstruction and physics performance $\quad 3$

4 Validation in beam tests $\quad 4$

5 Conclusion 5

\section{Introduction}

The discovery of the Higgs boson at the LHC by the ATLAS and CMS Collaborations has opened a new path for the search of physics beyond the standard model. The new scalar boson has been observed in several decay channels [1], in particular in the bosonic channels where its mass and spin-parity properties have been determined. Nevertheless, many pieces of the electroweak symmetry breaking sector still need to be better understood and require much more luminosity than the current phase of the LHC will deliver. This is, for instance, the case for the observation of rare Higgs decays (such as $H \rightarrow \mu \mu$ and $H \rightarrow Z \gamma$ ), to constrain the Higgs boson self-coupling via double-Higgs production, and to perform precise unitarity tests with vector boson scattering. High luminosities at the LHC will in addition extend the searches of new particles at high masses or low couplings.

The high-luminosity phase of the LHC (HL-LHC) is scheduled to start around 2026 and will accumulate an integrated luminosity of about $3000 \mathrm{fb}^{-1}$. The radiation-induced damages of the current ECAL and HCAL calorimeters in the endcaps of the CMS detector [2] will require their replacement with a radiation-hard technology. In addition to being resilient to a very high level of accumulated doses, the new calorimeter will have to face the pileup (PU) of up to 200 simultaneous proton-proton interactions. These requirements have driven the choice of the calorimeter technology used for this replacement. The High Granularity Calorimeter (HGCAL) [3] will have fine transverse and longitudinal segmentation and will be able to measure precisely the time of arrival of incoming particle showers, in order to disentangle contributions from many interactions.

The overview of the HGCAL detector is presented as well as its performance obtained in simulation. This performance has also been validated in several beam-test campaigns, which are summarised here. 


\section{HGCAL design overview}

The HGCAL is a sampling calorimeter composed of an electromagnetic (CE-E) and a hadronic (CE-H) section made of 28 and 24 layers of active material respectively. The electromagnetic part uses lead, copper, and copper-tungsten alloy as absorbers while copper and steel are used for the hadronic part. Silicon sensors are used in the region with the highest level of radiation, whilst plastic scintillator with on-tile silicon photomultipliers are used for the part of the CE-H where the radiation level is the lowest. The key parameters of the HGCAL are summarised in Fig. 1.

Surfaces of about $600 \mathrm{~m}^{2}$ of silicon sensors and $500 \mathrm{~m}^{2}$ of scintillator tiles are used. The silicon sensors are finely segmented into 0.5 and $1 \mathrm{~cm}^{2}$ cells with active thicknesses of $300 \mu \mathrm{m}, 200 \mu \mathrm{m}$ or $120 \mu \mathrm{m}$ depending on the region of the detector. These cell parameters are chosen such that the signal-over-noise ratio remains high enough for the measurement of MIP signals over the full lifetime of the HGCAL. Scintillator cells of $1^{\circ}$ and $1.25^{\circ}$ are used, arranged in a $\eta-\phi$ grid.

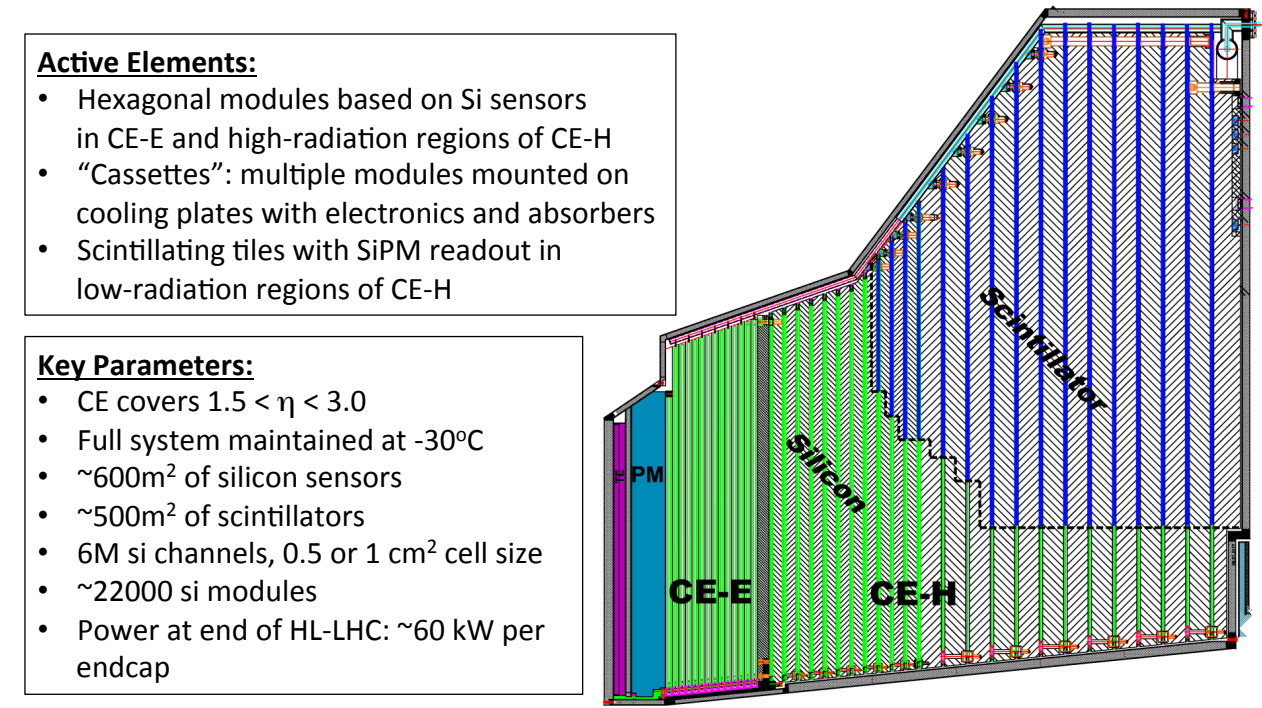

Electromagnetic calorimeter (CE-E): Si, Cu \& CuW \& Pb absorbers, 28 layers, $25 \mathrm{X}_{0} \& \sim 1.3 \lambda$ Hadronic calorimeter (CE-H): Si \& scintillator, steel absorbers, 24 layers, $~ 8.5 \lambda$

Figure 1. Schematic longitudinal cross section of the HGCAL calorimeter and its key parameters. The silicon and scintillator parts of the detector are highlighted in green and dark blue respectively.

For the CE-E part, silicon sensors are sandwiched between copper-tungsten baseplates and PCBs assembled into hexagonal modules, and mounted on both sides of a copper cooling plate. Stainless steel tubes inside the cooling plate carry two-phase $\mathrm{CO}_{2}$ to maintain the silicon sensors at a temperature of $-30^{\circ} \mathrm{C}$. Each cooling plate is covering an azimuthal angle of $60^{\circ}$ over the full radius of each layer. Plates of lead and stainless steel of the same dimensions cover the sensors and electronics. This assembly is called a "cassette", and 14 cassettes are assembled in a stack to form the CE-E. 
For the CE-H part, the absorber is made of full disks of stainless steel forming a rigid mechanical structure in which the active elements are inserted as cassettes of $60^{\circ}$. The $60^{\circ}$ cassettes are split in $30^{\circ}$ units to keep their size manageable. The first 8 layers are fully instrumented with silicon sensors, while the rest of the layers are made of scintillator tiles in the low pseudorapidity region and silicon sensors in the high pseudorapidity region. As opposed to the CE-E part, the detector modules are assembled only on one side of the copper cooling plate.

The front-end electronics are shared on two ASICs located on two different PCBs. The first ASIC, the HGCROC, is located on the sensor PCB and includes analog and digital parts reading out signals from up to 72 cells. The second ASIC, located on a different PCB, concentrates the data coming from several HGCROCs covering up to 6 modules and sends the data to the backend electronics on $10 \mathrm{~Gb} / \mathrm{s}$ optical links. A reduction of the data is performed to send trigger data at $40 \mathrm{MHz}$ to the backend electronics, while the full granularity data are stored in buffers in the HGCROC before being sent out at $1 \mathrm{MHz}$ after a latency of $12.5 \mu \mathrm{s}$. For the silicon part, the frontend electronics provides a charge measurement on a large dynamic range, from $0.2 \mathrm{fC}$ to $10 \mathrm{pC}$, as well as a precise timing information with an accuracy better than $100 \mathrm{ps}$ for pulses above $12 \mathrm{fC}$.

\section{Reconstruction and physics performance}

The highly granular data provided by the HGCAL, both in the lateral and longitudinal dimensions, as well as the precise timing information, provide detailed inputs to reconstruct efficiently events containing many PU interactions. This fine-grained information can be optimally used by particleflow algorithms to disentangle the showers from nearby particles and reconstruct their energy and shower properties with a high accuracy.

The energy resolution of electromagnetic showers is an important measure of the performance of the detector, in particular the value of its constant term, given the high energy particles expected in this high pseudorapidity region. The electromagnetic resolution is shown in Fig. 2 (left) for three silicon thicknesses. Constant terms below $1 \%$ are obtained, assuming an intercalibration of the cells at the level of $3 \%$. The procedure of intercalibration has been carefully studied to ensure that this $3 \%$ precision can be maintained throughout the lifetime of the detector.

The transverse size of electromagnetic showers is also an important characteristic of the detector, which has to be low enough to be able to disentangle close-by showers. It is shown in Fig. 2 (right) that a Molière radius of about $3 \mathrm{~cm}$ is obtained, with shower sizes below $1.5 \mathrm{~cm}$ in the first layers.

The particle identification is also of critical importance in events with a high hadronic activity. Electromagnetic and hadronic showers can be efficiently separated thanks to precise shower-width variables, improved by the capability of reconstructing the principal axes of the showers. The longitudinal dimension can also be used to separate the different development of electromagnetic and hadronic showers. The separation between electromagnetic and hadronic showers using the shower width in the radial direction and a measure of the shower length is shown in Fig. 3.

In addition, the availability of precise timing information will provide an additional powerful handle to remove contamination from PU showers. The time resolution per cluster below $20 \mathrm{ps}$ for cluster energies above $10 \mathrm{GeV}$ can be used to clean PU contaminations in hadronic jets and to discriminate bremsstrahlung photons from PU neutral pions in the clustering of electron energy 

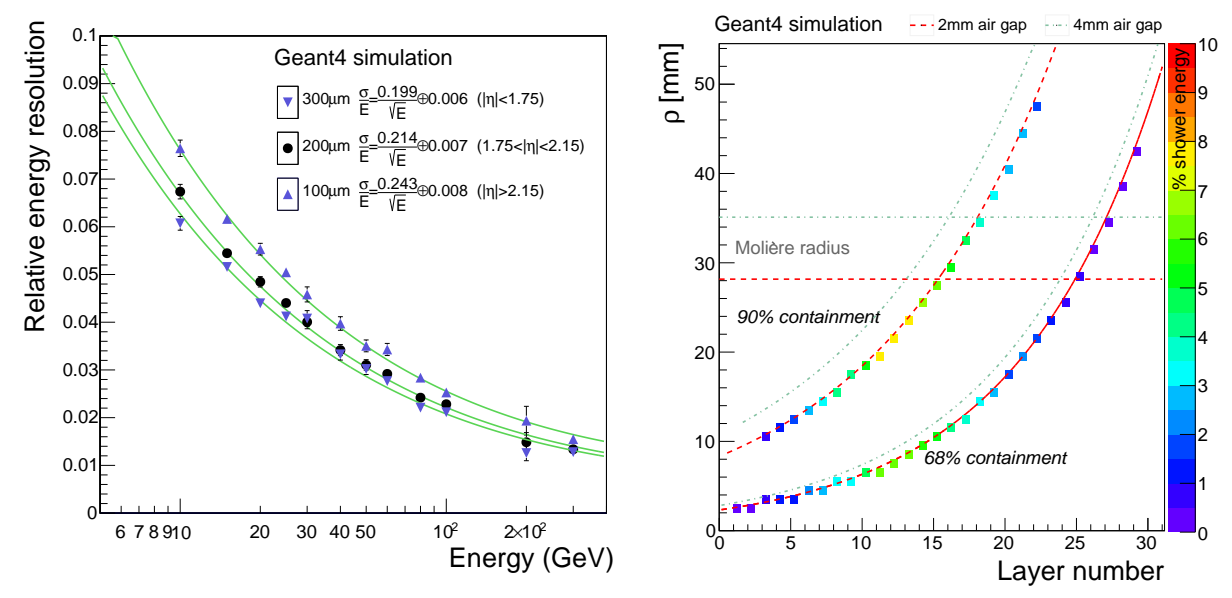

Figure 2. Left: electromagnetic energy resolution as a function of the energy for three different thicknesses of the silicon sensors $(100 \mu \mathrm{m}, 200 \mu \mathrm{m}$ and $300 \mu \mathrm{m})$. Right: $68 \%$ and $90 \%$ containment of electromagnetic showers in the CE-E, as a function of the layer. A simulation of the detector with Geant 4 has been used for both results.
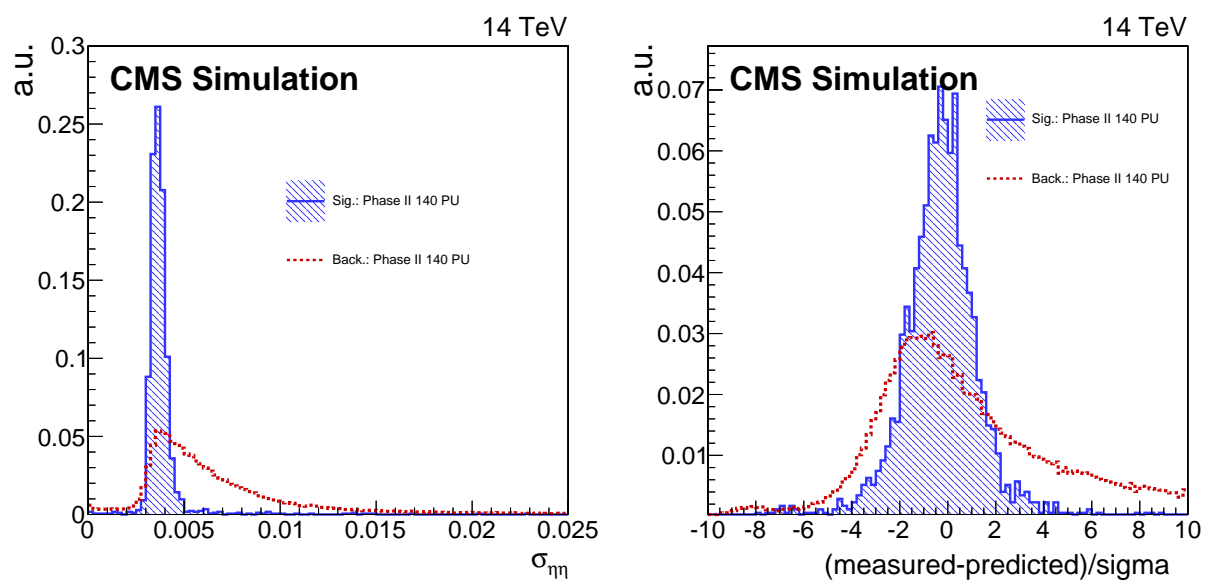

Figure 3. Distributions of the shower transverse width along $\eta$ (left) and of the compatibility of the measured shower length with the length expected for an electromagnetic shower (right). The distributions for true electrons from $Z$ decays (blue) and for hadronic jets reconstructed as electrons (red) are compared.

deposits. The timing information can further be used at the level of the full event to perform vertex triangulation, for instance to improve the $H \rightarrow \gamma \gamma$ mass reconstruction.

\section{Validation in beam tests}

Beam-test campaigns have been conducted with electrons, hadrons and muons of various energies, to validate the detector concepts and its simulation. Up to 20 modules have been put in beam lines at FNAL and at CERN to probe electromagnetic showers (up to $27 X_{0}$ ) and hadronic showers (up to $4 \lambda$ ). The noise was sufficiently low such that the MIP peak could be extracted with a signal-over-noise ratio of more than 7 (see Fig. 4 (left)) for the intercalibration of all the cells. The reconstructed showers have also been compared with simulated events. The simulation showed a 
very good agreement both in terms of energy resolution and in terms of shower shapes. As an example the distribution in data and simulation of the ratio of the energies in one ring and two rings of cells $\left(E_{7} / E_{19}\right)$ for hadron showers is shown in Fig. 4 (right).
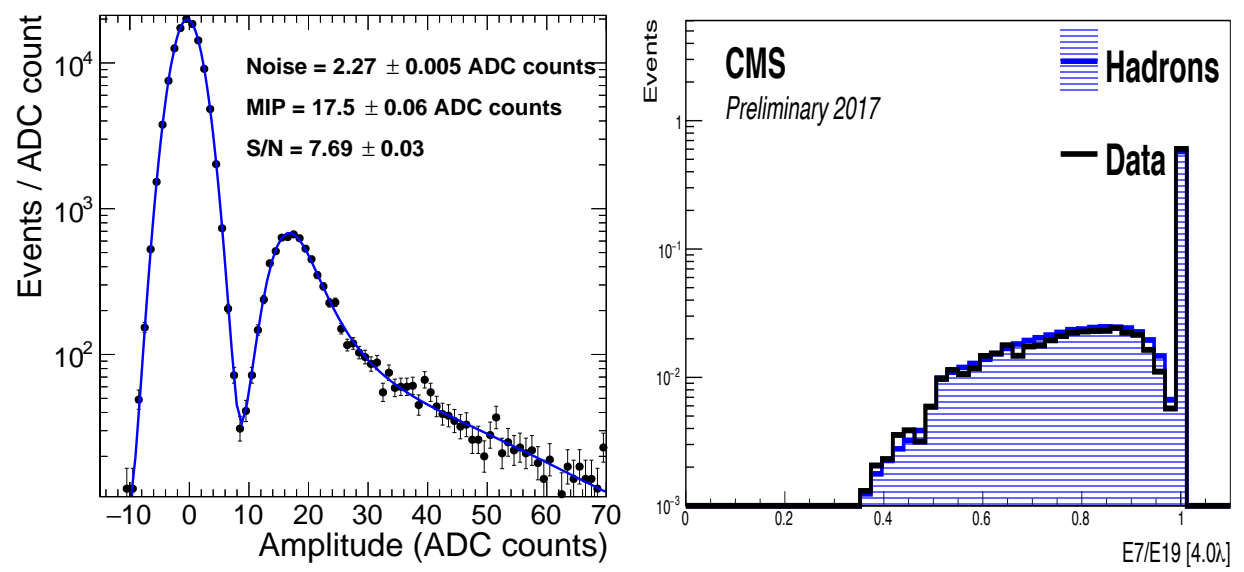

Figure 4. Left: distribution of the amplitude of the pulses in ADC counts for incident charged pions in a typical silicon cell. Right: Distribution of the ratio of energies in one ring $\left(E_{7}\right)$ and two rings $\left(E_{19}\right)$ of cells measured at a depth of $4 \lambda$ for incident charged hadrons.

\section{Conclusion}

The high-granularity calorimeter project HGCAL developed for the replacement of the current CMS endcap calorimeters is progressing on every front. Solutions for the mechanics, the electronics, and the reconstruction have been explored to deliver the best possible performance under the challenging pileup and radiation environment of the HL-LHC. The HGCAL will provide not only a measurement of energies but will also track the development of the showers and measure their time of arrival. It will pave the way to detectors that will run at future colliders.

\section{Acknowledgment}

The work of the author of this paper has been partly funded by the P2IO LabEx (ANR-10-LABX0038) in the framework "Investissements d'Avenir" (ANR-11-IDEX-0003-01) managed by the French National Research Agency (ANR).

\section{References}

[1] ATLAS and CMS Collaborations, Measurements of the Higgs boson production and decay rates and constraints on its couplings from a combined ATLAS and CMS analysis of the LHC pp collision data at $\sqrt{s}=7$ and $8 \mathrm{TeV}$, JHEP 08 (2016) 045

[2] CMS Collaboration, The CMS experiment at the CERN LHC, JINST 3 (2008) S08004

[3] CMS Collaboration, Technical Proposal for the Phase-II Upgrade of the CMS Detector, CERN-LHCC-2015-010, LHCC-P-008, CMS-TDR-15-02 (2015) 